\title{
Integrazione sociale in comunità rurali: il caso concreto della comunità hippy in
}

Ibiza

\author{
Simone Belli \\ Universitat Autònoma de Barcelona \\ simo.belli@tiscali.it
}

\begin{abstract}
Resumen
Abstract

Il compito della ricerca è quello di verificare attraverso

The aim of the study is to verify, by means of le interviste, se esiste una comunicazione tra hippy e interviews, if a communication between hippies and autoctoni nella isola di Ibiza negli Anni '60, epoca in cui natives in the island of Ibiza in the Sixties exists, when avvengono i primi insediamenti.Per far ciò ci siamo the first settlements took place. To achieve this goal, we avvalsi dell'uso dell'analisi del discorso (AD) e used Discourse analysis (DA) and mainly philosophy of principalmente della filosofia della analisi della Conversation-analysis (CA), in this way we have been conversazione (AC), in questo modo abbiamo potuto able to analyze the long periods of narration, the soanalizzare i lunghi periodi di narrazione, le cosiddette called life story.
\end{abstract}

life story, storie di vita.

Palabras clave: Análisis del Discurso, Emociones en Keywords: Discourse Analysis, Emotion in language, el lenguaje, historias de vida, comunidad hippy

life story, hippie community

\section{Introduzione}

Questa articolo estrae gli argomenti maggiormente significativi della suddetta ricerca, che ha come obiettivo principale la verifica dell'esistenza di comunicazione, tra due comunità differenti, hippy ed autoctoni, che occupano lo stesso spazio, l'isola di Ibiza, nello stesso periodo storico, gli anni sessanta. Tutto ciò verra effettuato in un'ottica discorsivista, secondo cui attraverso la comunicazione, è possibile la comprensione di quei processi cognitivi che con il linguaggio è possibile identificare.

Questo caso concreto, è particolarmente utile per utilizzare e mettere in pratica, sul campo, le teorie psico-sociali attuali. Vedremo com'è possibile adottare la Teoria dell'Identità Sociale di Tajfel e delle Rappresentazioni Sociali di Moscovici, secondo una visione completamente nuova, infatti si è utilizzato i particolari sviluppi che queste teorie hanno sviluppato, delle correnti alternative, riguardanti l'evoluzione discorsivista, che recenti autori hanno intrapreso. In cui l'azione principale è dettata dal 
linguaggio, dalla comunicazione. La parola come fatto. Sono state raccolte storie di vita come dimostrazione di questo, ed analizzate secondo l'ottica dell'Analisi del Discorso, concentrandoci sulla particolarità ed importanza dei singoli termini. Così da poter costruire un modello utile per esplicare il nostro caso.

Mi è sembrato opportuno, per rendere queste teorie maggiormente significative, lo studio di un caso concreto, dove la varietà delle situazioni e l'ampio sviluppo del tema, è terreno particolarmente fertile per queste concezioni discorsiviste. L'adozione di un caso concreto alla ricerca, ci è utile per dare un significato pratico alle teorie psico-sociali viste, cercando di adattarle al fenomeno studiato, così poter evidenziare le particolarità di questi innovativi approcci. Lo studio di questo particolare fenomeno è dettato principalmente dalla conoscenza del luogo, della lingua e di alcune persone che ci vivono, così da poter fare una ricerca sul campo in un ambito differente dal solito contesto in cui ci troviamo. L'isola d'lbiza, ancora poco conosciuta sotto questo aspetto, molte volte nascosto dalla "fauna" estiva che la occupa, ci è sembrata un fenomeno interessante per l'analisi, e fino ad oggi, poco studiato. Infatti, poche opere affrontano questo tema, uno studio approfondito di ciò che è successo sull'isola negli anni sessanta, non è ancora stato fatto. Quindi quale miglior occasione per essere tra i primi a svolgere una ricerca in questo contesto. Soprattutto nell'ottica in cui, questo studio va eseguito il più presto possibile, perché gli autoctoni e gli hippy che hanno partecipato a quel processo di integrazione in quegli anni, sono ormai gente di maggiore età, ed urge una raccolta di dati per questo fenomeno.

Ci si propone di dare significato, ai nuovi aspetti delle scienze sociali, soprattutto un maggiore utilizzo in diversi campi della Psicologia Sociale Discorsiva, non chiusa solo in quest'ambito, ma aperta a nuove possibilità di applicazione.

L'osservazione avviene seguendo una chiara e precisa concezione di base discorsivista, lasciando ampi spazi ai parlati-in-interazione, con ampi segmenti di life story, e cercando di non stravolgere il contenuto di una discussione, ma lasciandola nel suo contesto originario. Concentrandoci maggiormente sui processi descrittivi riguardante la comunicazione e l'integrazione tra le due culture.

\section{Psicologia discorsiva, Emozioni e memoria nelle narrazioni}

Il compito della ricerca è quello di verificare attraverso le interviste, se esiste una comunicazione tra hippy e autoctoni nella isola di Ibiza negli Anni ‘60, epoca in cui avvengono i primi insediamenti.

Per far ciò ci siamo avvalsi dell'uso dell'analisi del discorso (AD) e principalmente della filosofia della analisi della conversazione (AC), in questo modo abbiamo potuto analizzare i lunghi periodi di narrazione, le cosiddette life story, storie di vita.

La prima domanda che l'analista del discorso si deve fare è la stessa che si fa un qualunque ricercatore sociale responsabile: Che fenomeno sociale si sta cercando di chiarire? Che relazioni sociali si vogliono spiegare? II modo particolare in cui l'analista del discorso colloca la questione consiste nel domandarsi che relazioni sociali mantenute e perseguite attraverso il linguaggio si cerca di spiegare. 
Per l'AD, il linguaggio non esiste "nella testa", ma esiste nel mondo: il linguaggio è più una forma di costruzione di noi stessi. L'AD riconosce il mondo nel quale il parlante vive, un mondo dove il parlato produce degli effetti, riconosce i contesti.

L'AD assume il linguaggio sia come segnale di una realtà, sia come forma di crearla. Fa un uso del linguaggio come forma dinamica, ed è sensibile ai suoi effetti, non nel senso come reazione mentale transitoria, ma come effetto delle forme linguistiche.

La prima nozione di qualunque avventura nelle Scienze Sociali che ci consiglia Iñiguez (1994), è che questa deve essere persuasiva, significa che il ricercatore deve stabilire una relazione attiva con il lettore e tentare di mostrare come il ricercatore effettua una buona lettura del testo, ciò consiste più in un esercizio di negoziazione che di esposizione.

II ricercatore non deve difendere l'uso di test statistici, neanche deve realizzare speciali sforzi per evidenziare che base di interpretazione si sta usando, o difendere le sperimentazioni come un paradigma appropriato di ricerca. L'analista del discorso deve solo assicurarsi che il lettore comprenda ciò che sta succedendo.

Scettici per l'uso completo dell'Analisi della Conversazione, si è optato per considerare questa solo per il suo contenuto filosofico.

La prima cosa è l'aspetto emico, il fatto di non imporre al mio sistema delle categorie di analisi, ma faccio emergere le caratteristiche dai parlanti.

II secondo aspetto che andiamo a cogliere dall'AC, poco conosciuto, ma non per questo non importante, è il Membership Categorization Analysis (MCA), ossia l'analisi delle categorie di appartenenza ad un gruppo, dal fondatore dell'AC, Harvey Sacks.

Tutto il resto della AC non si conforme a questo genere di lavoro, in quanto I'AC ha un procedimento troppo meticoloso.

La relazione che possiamo individuare tra $A D$ ed $A C$ si tratta, secondo Iñiguez (1996, p.112), di un'operazione di inclusione. L'AC ha sviluppato un congiunto di procedimenti e regole straordinariamente utili per l'analisi delle azioni sociali, tecniche che hanno una solida fondamenta di base teorica che vanno dalla filosofia analitica fino all'etnometodologia, però ha alcuni problemi che stanno alla base del suo carattere evidentemente induttivo, in quanto suppone nella comprensione che il linguaggio è uno strumento che costruisce e regola le azioni sociali e il come possiamo analizzarlo, in base alla trascrizione letteraria delle conversazioni quotidiane. Invece l'AD come analisi procede in modo deduttivo, in quanto possiamo definire il mondo in base all'analisi interpretativa dei testi. Quindi il trovare una giusta relazione tra questi due tipi di analisi ci porta a sviluppare una tecnica/interpretazione utile per la nostra ricerca.

Le life story che ci siamo trovati ad analizzare in questa ricerca, sono delle vere e proprie narrazioni. La costruzione narrativa del mondo, vista da una approssimazione psicologica sociocostruzionista, che come scopo ha, l'azione sociale, e il suo carattere dilemmatico e politico, come ci dicono Teresa Cabruja, Lupicinio Iñiguez e Félix Vàzquez (2000).

Così la narrazione è strettamente legata all'azione più che all'elaborazione di una storia, un relato o una testimonianza. Le narrazioni permettono la coordinazioni di azioni sociali. 
Le narrazioni sono quegli strumenti attraverso dei quali diamo un senso al nostro mondo, costituendolo come significato per noi stessi. La narrazione è uno dei principali attrezzi per la costruzione della realtà simbolica.

Nel libro di Felix Vàzquez, La memoria como acciòn social, forse uno dei più bei libri riguardo alla memoria considerata come un processo dinamico, attivo che si costruisce, proprio come un'azione sociale. Vàzquez sostiene che la memoria sociale può essere considerata come lacci deboli o lacci invisibili, cioè una componente indissociabile che assorbe gran parte dei fenomeni e processi sociali e che possibilitano la continuità del senso sociale.

Per Vàzquez, l'importanza dello studio della memoria e del ricordo sociale risiede nel suo carattere di processi che contribuiscono, definendo e articolando, l'ordine sociale. Vivere in società implica avere memoria e avere ricordo.

Ci troveremo nelle nostre storie di vita delle memorie condivise, in quanto relazioni multiple, vissute contemporaneamente da un gruppo sociale, da delle comunità differenti e quindi il processo di memoria è stato sviluppato in maniera collettiva e condivisa. Vàzquez sostiene che quando facciamo memoria, siamo noi che definiamo se effettivamente, stiamo parlando della nostra memoria o della memoria di altri, se si producono errori o non errori che ricordiamo, se produciamo inganni, etc.

La memoria si arricchisce sempre più di particolari, per rendere più credibile discorso, che è possibile vengano creati dopo una nostra personale elaborazione. Infatti la memoria può contare solo su certi fatti o azioni, che nel momento in cui le abbiamo vissute ci sono sembrate maggiormente significative, sono pochi questi fatti certi e realmente accaduti che possono essere presenti nei nostri discorsi per molti anni, pero col tempo si confondono con fatti non reali e che noi li percepiamo come reali. Quindi la memoria a questo punto viene influenzata dalle emozioni, sono loro le principali attrici dei nostri discorsi.

Soprattutto quando si parla di memoria.

\section{Teorie fondamentali della Psicologia Sociale contemporanea}

Ci siamo chiesti se è possibile constatare che le due comunità studiate possano essere definite tali o no, in quanto gli autoctoni sicuro che si, sono una comunità, ma gli hippy?

In quanto già lo erano in precedenza, una comunità, o sono arrivati ad Ibiza individualmente, con delle scelte autonome e non collettive?

Queste sono le domande principali che ci siamo posti prima di poter lavorare per studiare questo caso. Infatti senza una risposta concreta a ciò, difficilmente potremo venire a capo di qualcosa.

Si tratta di scoprire, se si tratta di una integrazione tra le due comunità, sempre se così si possano chiamare, oppure se ci sono state tensioni, a livello percettibile della popolazioni, giustamente uno stadio prima della situazione conflittuale, in quanto non possiamo parlare di un conflitto vero e proprio.

Se ciò è avvenuto, se l'immigrazione collettiva è avvenuta e tutto ciò che possa scaturire da questi avvenimenti, si può comprenderlo solo dalle interviste effettuate. 
"Invece di vedere quali identità le persone abbiano, gli analisti della conversazione si concentrano sul se, quando e come le identità vengono usate" (Widdicombe, 1990)

Esistono poche analisi dirette ai possibili punti di intersezione tra le teorie dell'identità sociale a proposito di cognizione ed azione sociale e la prospettiva dell'analisi del discorso. Oltre l'interesse dei teorici dell'identità sociale per l'autopresentazione strategica (collettiva), e la funzione degli stereotipi sociali e l'attribuzione nei contesti di argomenti relazionati con la giustizia sociale e la legittimità, fino a poco tempo fa i teorici dell'identità sociale realizzavano le loro ricerche unicamente nell'ambito del laboratorio. Solo negli ultimi tempi alcuni di loro, tentarono di esplorare i loro interessi teorici impiegando dati che provenivano dal linguaggio naturale.

\section{La Ricerca}

Ora cercheremo di adattare tutte le varie teorie precedentemente viste con il materiale raccolto sul campo. Si vede com'è possibile utilizzare l'Analisi del Discorso e le diverse Teorie dell'Identità Sociale su casi concreti, come le due comunità che ci troviamo a studiare.

Attraverso le life story, vediamo come il concetto di interculturalità veniva elaborato dai locali, gli autoctoni.

Per analizzare le interviste raccolte, si è optato per l'analisi del contenuto tematico, perché è stato il metodo più soddisfacente per l'elaborazione di questa ricerca.

Molte volte l'analisi del discorso non è il metodo più idoneo per procedere ad analizzare dati testuali, infatti, bisogna sempre porsi la domanda: "Che voglio fare con questi dati?". Possiamo scoprire che l'analisi che realmente necessitiamo, sia quella del contenuto. Come sostiene Vazquez (1996), esistono mezzi adeguati per fini specifici.

Il funzionamento dell'analisi del contenuto categoriale è relativamente semplice. Partendo da dati testuali, si scompone il testo in unità, così da poter raggruppare in categorie, seguendo il criterio di analogia.

Vazquez sostiene che uno strumento è valido quando misura ciò che è destinato a misurare. Rispetto all'analisi del contenuto, diciamo che questo è valido se i risultati ottenuti sono equiparabili ad altri risultati ottenuti da analisti differenti da noi, utilizzando gli stessi procedimenti.

La validità dell'analisi del contenuto deve essere esaminata se le trasformazioni operate nei dati durante il processo di analisi (dai "dati bruti" ai "dati utili") hanno corrispondenza con il significato che gli si attribuisce nella produzione o nell'emissione degli stessi. In altre parole, se i risultati delle analisi corrispondono con i fenomeni ed i processi reali nel contesto sociale dove questi dati si producono. Questo implica che tutti i procedimenti utilizzati nell'analisi del contenuto devono essere sensibili al contesto sociale dal quale provengono e si ubicano.

Esistono diversi metodi per accreditare la validità. Nonostante ciò, per i nostri interessi solo facciamo riferimento alla validazione per giudizio. In sintesi, questo consiste che differenti persone, seguendo le regole assegnate per l'esecuzione dell'analisi trattano di arrivare agli stessi risultati a cui siamo arrivati noi. La confermazione della validità verrà data per la ratificazione dei risultati e delle conclusioni. Al 
contrario, si considererà che il processo non riunisce i requisiti minimi di validità se i giudizi non raggiungono gli stessi risultati che precedentemente descriveva il seguimento del processo elaborato.

II materiale raccolto sull'isola d'Ibiza è composto da: life story, documenti personali, diari, foto, articoli tratti da quotidiani e da siti internet. Così da avere una visione generale delle due comunità e poter disporre di più punti di vista.

Prima della mia partenza per Ibiza, abbiamo preparato un modello per le interviste, o come lo chiama Iñiguez, "el guion de las entrevistas", che tradotto letteralmente è il copione da seguire per le interviste. Questo "guion" è stato creato in base ai quattro criteri di Pujadas Muñoz (1992), riguardo alle storie di vita nelle scienze sociali:

1. Elaborare uno schema teorico della ricerca che spieghi chiaramente quali sono le ipotesi del lavoro iniziale;

2. Giustificare metodologicamente il perché si è eletto il metodo biografico;

3. Delimitare con maggior precisione possibile, l'universo di analisi (comunità, etc.);

4. Spiegare i criteri di selezione degli intervistati

Lo schema teorico della ricerca nel nostro caso, si compone in tre punti fondamentali:

1. Obiettivi principali;

2. Obiettivi specifici;

3. Strumenti e concetti analitici al servizio della ricerca.

Per prima cosa, definiamo l'obiettivo principale della ricerca:

verificare se esiste una comunicazione tra hippy ed autoctoni nell'isola di Ibiza negli anni '60, così da poter individuare ed analizzare le seguenti fasi (obiettivi specifici):

1. Vedere se si possono definire due comunità questi due gruppi di persone (gli autoctoni si, però gli hippy quando hanno costituito una comunità? Prima già lo erano o è stata una migrazione di carattere individuale?). Conseguentemente, potremo adottare le varie teorie, "processi di differenziazione e conflitti intergruppali" di Turner, "paradigma del gruppo minimo" di Tajfel, "comportamento intragruppo e intergruppo" di Tajfel, " come le identità sociali si concentrano e vengono usate, il sé, il quando e il come" di Widdicombe.

2. E' un caso di integrazione di due comunità, o di conflitto, tensioni?

Per lo sviluppo della ricerca, ci siamo serviti di strumenti "tecnici", di concetti analitici, suddivisi in questo modo:

1. la memoria nella narrazione (Bruner, Vazquez); le emozioni nella narrazione (Edwards);

2. la teoria delle rappresentazioni sociale fondata sull'azione, la comunicazione, costruita nel parlato e nel testo, che porta l'indagine sui processi verbali (Potter, Edwards, Billig, Wetherell, lbañez). 
3. "Caja herramientas conceptuales" di Iñiguez, contenente l'analisi del discorso e la filosofia dell'analisi della conversazione.

Si è scelto l'analisi delle life story, storie di vita, perché vogliamo capire principalmente che è successo quando sono arrivati i primi hippy sull'isola, ed in che modo essi hanno influito sulla comunità locale. Quindi, la cosa più importante che deve emergere dalle interviste, sono le relazioni tra hippy ed autoctoni.

II modello delle interviste deve essere differente, tanto nella forma, come nel modo, tra hippy ed autoctoni, perché le comunità sono agli antipodi quasi, anche se pensano lo stesso, però per sapere qualcosa di importante, dovremo persuadere nel parlare con sfumature diverse (come ad esempio i temi di sesso e droga). Realizzare le domande in maniera totalmente differente:

Domande per gli hippy:

$1^{a}$ pregunta : Me hablas un poco de él/ella y porquè ha tomado la decision de venir a lbiza y no a otro lugar?

$2^{\mathrm{a}}$ pregunta: Como se relacionó con la gente de aquí (los autóctonos), qué cosa le gustò y qué cosa no le gustó de aqui?

$3^{a}$ pregunta: Me gustaría saber como estaba y está todavía, organizada su vida aquí.

Domande per gli autoctoni:

$1^{\text {a }}$ pregunta: Ha cambiado mucho Ibiza en los ultimos años...

$2^{a}$ pregunta: Que piensa de los hippy que han llegado aqui, que le parece? Cree que se han integrado o que? Algunos de estos cambios tienen que ver con los hippy?

$3^{a}$ pregunta : Todo eso que ha pasado, cree que esta bien o no para la Isla ?

Molto importante è il tono assunto nelle interviste per differenziarsi tra hippy ed autoctoni, perché ad un autoctono non possiamo parlargli in maniera diretta di droghe o di sesso, come si potrebbe parlare ad un hippy, bisogna essere più discreti. Per assumere quest'atteggiamento, dovremo strutturare le domande sempre in questa particolare forma, iniziando con se dice...; dicen la gente...; han dicho que...; que piensa de....

Le interviste sono state raccolte tutte sull'isola d'Ibiza, in quattro lingue diverse: castigliano, inglese, italiano ed ibicienco.

Il campione degli intervistati è costituito da 15 individui, suddivisi in sette autoctoni ed otto hippy, d'età e di provenienza diversa. Anche per gli autoctoni, si è cercato di intervistare gente di diversa estrazione sociale e che vive in diverse zone dell'isola.

Fondamentale alcune volte, l'intervento dei mediatori culturali, soprattutto durante le interviste in cui la lingua parlata era l'ibicienco.

Mi è sembrato opportuno inserire nelle tabelle anche il luogo, dove sono avvenute le interviste, così è facili capire il contesto in cui mi sono trovato a lavorare, mercatini hippy, bar, per la strada, in case private, etc. Tutto ciò importantissimo, per intendere come il contesto ha influito sulle interviste. 


\begin{tabular}{|c|c|c|c|c|c|c|c|c|}
\hline & Nome & $\begin{array}{l}\text { Anno } \\
\text { arrivo ad } \\
\text { lbiza }\end{array}$ & Occupazione & Provenienza & $\begin{array}{l}\text { Dove risiede } \\
\text { nell'isola }\end{array}$ & $\begin{array}{l}\text { Presenza del } \\
\text { mediatore } \\
\text { culturale }\end{array}$ & $\begin{array}{l}\text { Luogo } \\
\text { dell'intervista }\end{array}$ & Lingua \\
\hline 1 & M. & 1972 & Musicista & Perù & Sant'Eulalia & Joan Noguera & $\begin{array}{l}\text { Mercatino di Es } \\
\text { Canà }\end{array}$ & Castigliano \\
\hline 2 & A. & 1965 & Artigiana & Inghilterra & Parte Nord & Joan Noguera & $\begin{array}{l}\text { Mercatino di Es } \\
\text { Canà }\end{array}$ & Castigliano \\
\hline 3 & Ma. & 1968 & Artigiano & Argentina & Parte Nord & - & $\begin{array}{l}\text { Mercatino di Es } \\
\text { Canà }\end{array}$ & Castigliano \\
\hline 4 & C. P. & - & Pittrice & Madrid, Spagna & Sant'Eulalia & - & Casa propria & Castigliano \\
\hline 7 & J. B. & 1976 & Fotografo & Argentina & Sant'Antonio & Pau Marimon & $\begin{array}{l}\text { Cafè del Mar, St. } \\
\text { Antonio }\end{array}$ & Castigliano \\
\hline 10 & S. C. & - & Pittrice & Milano, Italia & Porteinax & $\begin{array}{l}\text { Pau } \\
\text { Marimon }\end{array}$ & Casa propria & Italiano \\
\hline 13 & $\begin{array}{l}\text { T.K e suo } \\
\text { marito Cr. }\end{array}$ & $\begin{array}{l}1973, \\
1975(C)\end{array}$ & $\begin{array}{l}\text { Artista } \\
\text { Scrittore (C) }\end{array}$ & $\begin{array}{l}\text { Salisburgo } \\
\text { Munich (C), } \\
\text { Germania }\end{array}$ & San Carlos & - & Casa propria & $\begin{array}{l}\text { Inglese, } \\
\text { Castigliano }\end{array}$ \\
\hline 15 & G. e Mi. & $\begin{array}{l}1969, \\
1969(\mathrm{M})\end{array}$ & $\begin{array}{l}\text { Capitano di } \\
\text { barche } \\
\text { Pittrice (M) }\end{array}$ & $\begin{array}{l}\text { Argentina } \\
\text { Madrid (M), } \\
\text { Spagna } \\
\end{array}$ & Città di Ibiza & Joan Noguera & Casa propria & Castigliano \\
\hline
\end{tabular}

Tabella Interviste Hippy 1

\begin{tabular}{|c|c|c|c|c|c|c|}
\hline & Nome & Occupazione & \begin{tabular}{|l|} 
Dove risiede \\
nell'isola \\
\end{tabular} & \begin{tabular}{|l|} 
Presenza del \\
mediatore culturale
\end{tabular} & \begin{tabular}{|l} 
Luogo \\
dell'intervista \\
\end{tabular} & Lingua \\
\hline 5 & $\begin{array}{l}\text { Anziana } \\
\text { Signora }\end{array}$ & Pensionata & Sant Josep & Joan Noguera & Casa propria & Castigliano \\
\hline 6 & P. & Pensionato & Sant Josep & Joan Noguera & $\begin{array}{l}\text { Scalinata di Sant } \\
\text { Joseph }\end{array}$ & $\begin{array}{l}\text { Castigliano, } \\
\text { Ibicienco }\end{array}$ \\
\hline 8 & F. R. & $\begin{array}{l}\text { Ex tassista, ora } \\
\text { in pensione }\end{array}$ & Sant Jordi & Pau Marimon & Casa propria & Ibicienco \\
\hline 9 & J. T. & $\begin{array}{l}\text { Docente } \\
\text { universitario }\end{array}$ & Buscastell & Pau Marimon & Casa propria & $\begin{array}{l}\begin{array}{l}\text { Castigliano, } \\
\text { Ibicienco }\end{array} \\
\end{array}$ \\
\hline 11 & M. R. & Bottegaia & Sant Jordi & Pau Marimon & $\begin{array}{l}\begin{array}{l}\text { Ippodromo di Sant } \\
\text { Jordi }\end{array} \\
\end{array}$ & \begin{tabular}{|l|} 
Castigliano, \\
Ibicienco
\end{tabular} \\
\hline 12 & J. R. & $\begin{array}{l}\text { Proprietario } \\
\text { Ippodromo }\end{array}$ & Sant Jordi & Pau Marimon & $\begin{array}{l}\text { Ippodromo di Sant } \\
\text { Jordi }\end{array}$ & Ibicienco \\
\hline 14 & M. & Proprietaria bar & Santa Agnès & Joan Noguera & Bar di Santa Agnés & Ibicienco \\
\hline
\end{tabular}

Tabella Interviste Autoctoni 1

Una volta trascritte le interviste, abbiamo individuato cinque macroargomenti utili per la ricerca, scartando molte categorie poco interessanti per questo lavoro, ma degni d'attenzione per altre ricerche. La suddivisione è stata per ordine tematico, che rispecchiano i vari quesiti che si pone questa tesi.

Nel primo possiamo cercare di analizzare la parte in cui ci sono le varie identità sociali in gioco, soprattutto un interessante aspetto della terminologia usata per descrivere ed identificare i due gruppi, per poi spostarci sugli argomenti fondamentali che questa ricerca si occupa di ottenere, cioè se esisteva, ed esiste tuttora, comunicazione ed integrazione tra le due comunità.

Nel secondo invece, possiamo capire il perché l'isola d'lbiza sia così importante per gli hippy, il motivo per cui è definita un paradiso perduto, quali qualità nasconde quest'isola delle Baleari rispetto ad altre isole.

Il terzo macroargomento contiene una sorta di motivazioni riguardanti l'essenza d'essere hippy, il perché queste persone hanno cambiato vita e come la situazione è evoluta dagli anni sessanta ad oggi. 
Nel quarto ci troviamo ad analizzare il caso emblematico di Ca n'Aneta, un semplice bar di "pueblo" divenuto importante per il movimento hippy.

Il quinto capitolo è un'insieme di luoghi comuni che la gente ha, a proposito degli hippy, e si cercherà di sfatarli in base alle interviste raccolte.

\section{Life Story d'Ibiza}

\subsection{Comunicazione ed integrazione}

L'importanza che tiene una definizione, il significato di una parola, credo sia l'elemento fondamentale per riscontrare una rappresentazione sociale.

Tutto questo, è ancora più facile riscontrarlo in un'epoca passata rispetto alla nostra, poiché nella società attuale, ormai alcune barriere di pregiudizi e di stereotipi sono state abbattute.

Altro fattore fondamentale per la creazione di questi stereotipi è il luogo preciso dove ciò è avvenuto, l'isola d'Ibiza. L'isola, con poche relazioni con la penisola europea, quindi per nulla influenzata dalla cultura europea.

Un'isola in cui il commercio non era sviluppato, la gente viveva con le cose che loro stessi producevano. Una rete di relazioni chiusa e con scarsa comunicazione con l'altro, con il non-ibicienco.

Molte visioni dell'altro, danno una sensazione di positività nel giudizio, alcune invece sono abbastanza dure, con giudizi irremovibili.

Soprattutto su alcuni argomenti ci sono delle forti contraddizioni, uno può essere quello dei soldi, abbastanza caro agli ibicienchi, ma quasi per nulla importante per gli hippy, che anzi, scappavano da queste città consumistiche per andare dove il denaro non aveva quell'importanza.

Non avevano soldi, non potevano comprarsi macchine nuove, e nemmeno molto cibo, così erano derisi e giudicati come dei poveri individui che non hanno niente di meglio da fare, che vivere sull'isola, in una situazione d'apparente povertà. E' così che la maggioranza degli autoctoni, credeva fossero gli hippy.

Gli autoctoni erano dei gran lavoratori, che basavano tutto sulla produzione, sulle risorse agricole, ed il benessere della propria casa, della propria famiglia, e vedersi arrivare questa gente che non lavorava, che non mangiava molto, che oziava tutto il giorno, era qualcosa che non potevano concepire. Tutto il mondo del contadino ibicienco era attaccato ai beni materiali, alla terra, alle cose, alla casa.

La storia d'lbiza è anche la storia dell'invasione turistica. Pure gli hippy sono tra quelli che hanno invaso l'isola, pero in particolar differente rispetto ai turisti, loro si sono stazionati ed hanno vissuto in stretto contatto con la gente locale.

Gli hippy avevano apportato dopo il loro arrivo, una risorsa alternativa, un qualcosa di nuovo, una nuova concezione della vita, in cui il turista ne andava alla ricerca per sfuggire dalla propria routine quotidiana almeno per pochi giorni l'anno, il tempo che durava la vacanza. 
Chiaro che gli abitanti dell'isola non hanno capito immediatamente questa apportazione, infatti, vedevano i turisti come chi portava a loro del denaro e gli hippy invece, come chi sullisola non portava una ricchezza economica. Ma col tempo hanno capito che pure gli hippy apportavano qualcosa di buono per l'isola, poiché si creava un'atmosfera particolare, una filosofia di vita, che il turista cercava, anche per scappare dai soliti stereotipati villaggi vacanze.

Una cosa è certa, che nemmeno gli hippy erano gente senza denaro, "squattrinati fannulloni", di fatto la maggioranza erano "figli di papà", laureati, che cercavano una vita alternativa rispetto a quella che avrebbero vissuto in una grande città.

In un'epoca in cui tutti lasciavano i piccoli paesi rurali dell'isola, per andare a vivere nelle città, loro lasciavano le metropoli, per cercare di vivere una vita più sana ed equilibrata nell'isola, e questo gli autoctoni difficilmente se lo spiegavano. Non riuscivano a capire il perché i propri figli lasciavano le loro case contadine ed il lavoro nei campi per trasferirsi nei nuovi appartamenti cittadini dell'isola, invece questi "capelloni" volevano vivere in case nascoste nel campo, senza elettricità e senza acqua corrente, ed imitare il lavoro del contadino.

Cominciarono a vederli come qualcuno che in qualche modo, anche se con modalità diverse, cercava di condurre una vita simile alla propria, a quella dei contadini. Quindi, in definitiva, li compresero e li accettarono.

Secondo gli hippy, l'ibicienco e l'isola d'Ibiza, sono andati mutandosi, di pari passo, in peggio negli ultimi quaranta anni.

L'opposta visione, che ci danno gli ibicienchi degli hippy, è profondamente diversa, e molte volte in profondo contrasto, si spazia dal buonismo assoluto al profondo disinteresse per l'altra cultura.

Però in generale non emerge dell'odio nei confronti dell'hippy, non c'è razzismo, non c'è intolleranza.

Pure il periodo storico in cui ci troviamo, la Spagna degli anni '60, non è certamente paragonabile all'attuale Spagna, quella di Zapatero, liberale, dai matrimoni gay. Ci si trovava nel bel mezzo della dittatura franchista, gli ibicienchi non hanno vissuto il sessantotto, come d'altronde, nemmeno il resto degli spagnoli, ma solo le numerose guerre civili che hanno devastato negli anni antecedenti l'intera Spagna. A differeneza di questi giovani, provenienti dalle grandi città europee, dove il sessantotto era vivo, vissuto nelle strade, voluto da questi ragazzi, e che trasferendosi ad Ibiza portavano avanti in qualche modo questi ideali, erano i testimoni di quel particolare periodo storico, ed arrivati sull'isola erano abbastanza scioccati da come la Spagna non era stata influenzata da questa corrente, da come il sessantotto non fosse arrivato. Ma tutto ciò non era abbastanza considerato dai giovani hippy che, in definitiva, non erano interessati alla situazione politica spagnola, e nemmeno erano interessati ad interagire con la popolazione locale per poter chiarire questa situazione, l'unica cosa che chiedevano e che volevano era il vivere la naturalezza, in completa libertà, senza danneggiare nessuno, e senza molestare gli abitanti dell'isola.

La comunicazione è la prima forma di scambio tra una persona e l'altra. Questa può essere caratterizzata da diverse modalità, ma essenzialmente la cosa importante è lo scambio verbale vero e proprio che avviene tra gli individui. L'importanza che esiste nel sapere se esisteva comunicazione o no, negli anni ' 60 , tra le due comunità, è fondamentale per questa ricerca, perché da ciò è possibile identificare che tipo d'integrazione è esistita, come si è formata e in quale lingua è avvenuta questa comunicazione. 
Effettivamente il problema della lingua, è uno dei dilemmi principali quando si parla di scambio linguistico tra hippy ed autoctoni. La lingua parlata dagli autoctoni è l'ibicienco, un catalano di forma dialettale, con molte parole usate solamente sull'isola, invece gli hippy parlano perlopiù l'inglese ed il castigliano.

La barriera della lingua si può superare se la gente vuole, è questa la cosa fondamentale. La volontà di una persona nel comunicare con l'altra, va al di là dell'idioma. Infatti, a Ibiza è avvenuto ciò, sono molti i fattori che a reso possibile quest'abbattimento della barriera dell'idioma.

Quindi da entrambi le parti c'era comunicazione, una buona comunicazione, e questo era senz'altro un buon punto di partenza per iniziare una integrazione. Tutto ciò accadeva negli anni sessanta, ora la situazione è decisamente cambiata, tra hippy ed autoctoni non esiste più questa comunicazione spontanea, tutto è diventato molto più complicato, ora non ci sono più solo hippy ed autoctoni, perchè si sono aggiunti una marea di turisti, ci sono i finti hippy, i figli degli autoctoni, e numerose altre sottocategorie che rendono sempre più difficile la creazione di una comunicazione spontanea.

Ora ci troviamo di fronte l'argomento cruciale della ricerca, giacché possiamo vedere se tutto ciò finora visto ed analizzato, ci permette di capire, se sull'isola d'lbiza negli anni sessanta, esisteva veramente integrazione tra autoctoni ed hippy.

Innanzitutto per integrazione, ci riferiamo ad una buona convivenza tra le due popolazioni, che si rispettano reciprocamente ed hanno instaurato una perfetta comunicazione, in cui non esistono seri conflitti, e le poche tensioni avute sono state superate, così da aver migliorato la forma di vivere insieme.

Il rispetto è un ottimo presupposto per una felice convivenza innanzitutto, ma il rispetto è solo un fattore di buona educazione, e ciò non significa che le due culture cercano di avvicinarsi. Non è possibile che un individuo di una popolazione diventi individuo dell'altra, e viceversa, così da avere un'integrazione completa, e dove non è possibile distinguere i due gruppi. Quest'integrazione completa non è avvenuta sull'isola, poiché i due gruppi di persone sono molto differenti tra loro, e sarebbe impossibile che abbiano cose in comune. L'unica cosa che hanno in comune, è l'amore per l'isola, l'amore per la terra ed il mare, queste sono le uniche cose che li accomunano, per tutto il resto sono profondamente differenti, diversissime mentalità, diversi comportamenti, storie di vita profondamente divergenti, sarebbe assurdo se fosse accaduta quest'integrazione completa.

Però il contatto tra hippy e contadino mutò, si trasformò, le cose non furono più uguali a prima, è un cambiamento che doveva accadere, in quanto importantissimo per la convivenza fra le due diverse culture. Non è possibile che le due culture si possano mischiare, ciò che invece è possibile, è che s'influenzino a vicenda, rispettandosi, capendo le proprie diversità e quelle altrui, così oltre che ad una felice convivenza, è possibile che le due culture si comprendano ed imparino cose nuove, utili alla propria esistenza.

E' diversissimo il modo di vivere delle due comunità, soprattutto quello di intendere l'isola, sono due aspetti in contrapposizione, per un gruppo l'isola è il duro lavoro della terra, per l'altro gruppo, l'isola è il posto dove poter essere se stessi, dove poter conoscere altre culture. Tutto ciò rende impossibile una forma d'integrazione dove ambedue i gruppi condividano gli stessi spazi, gli stessi luoghi, facciano le medesime cose e si relazionano con la medesima gente. 
Ancora adesso quando si parla di integrazione, molti rispondono che tuttora non sono realmente integrati, in parte ciò è vero, come ho detto prima, molti sono i gruppi chiusi, con poche aperture verso l'esterno, l'esterno in questo caso sono gli altri, che sono nell'isola, e non il turista, lo straniero.

C'è una più che rispettuosa convivenza, ma non una vera e propria integrazione, non è avvenuto uno scambio tra le due culture, questo sicuramente perchè nessuno lo voleva.

\subsection{Nascita e morte di un paradiso perduto}

Ibiza è cambiata, è un'opinione comune, sia di autoctoni che di hippy, tutti ripetono sempre la stessa frase. Pare che il cambiamento sia inevitabile, però l'essenza dell'isola rimane.

Alcuni sostengono che ciò è dovuto al troppo cemento, alla mala gestione politica, ai troppi turisti, al cambiamento di mentalità degli stessi ibicienchi, le colpe sono da destinarsi a chiunque, e nessuno sa dire se ciò corrisponde a verita o meno, in una cosa però tutti sono d'accordo, l'isola non è più la stessa.

Non è più la stessa, anche perché nel periodo in cui gli hippy l'avevano conosciuta, avevano vent'anni, ed a vent'anni quasi qualunque luogo è bello, ora invece è la vecchiaia che avanza, e un'isola può anche essere scomoda, non ospitale come un tempo, si scoprono i difetti che prima non esistevano, e così si dimentica tutte quelle cose che rendevano Ibiza unica negli anni sessanta.

Chi può dirlo se Ibiza ne avrebbe guadagnato, se tutta quell'ondata di turisti non fosse mai arrivata, è possibile pure che ne avrebbe perso, in quanto molta gente non avrebbe conosciuto l'isola. Alcuni potranno sostenere che un paradiso perduto, è perduto a volte, perchè lo conoscono in pochi. Finchè c'erano pochi turisti benestanti e alcune comunità hippy che frequentavano l'isola, Ibiza ne guadagnava, ora invece la situazione è cambiata.

\subsection{Perche' ibiza e' diventata l'isola libera?}

Vediamo ora le cause ed i perchè molta gente da tutte le parti del mondo è arrivata ad Ibiza, che cosa li ha spinti a lasciare il proprio Paese e cercare un'altra alternativa di vita.

In quegli anni, il periodo che va dai sessanta ai settanta, c'erano ancora guerre da combattere, regimi da abbattere, ed il mondo, anche se sono passati solo pochi anni, non assomigliava a quello che vediamo ora.

Negli Stati Uniti, i giovani, per paura di essere chiamati alle armi, e di conseguenza, andare a combattere nella guerra del Vietnam, scappavano, lasciavano le proprie case, alcuni vennero ad Ibiza, si rifugiarono sull'isola.

Tutti questi hippy, una volta arrivati ad Ibiza, come vivevano, come si guadagnavano i soldi per pagarsi l'affitto, il cibo, l'alcol e le droghe, per le feste che organizzavano ogni notte?

Alcuni ricevevano uno stipendio dai propri genitori, ogni mese gli inviavano per posta, una discreta quantità di denaro, con la quale potevano vivere sull'isola, alcuni meno fortunati, si arrangiavano con piccoli lavoretti di artigiania, da vendere nei mercatini o al porto di Ibiza, per i turisti di quell'epoca.

Gli autoctoni faticavano a comprendere questi lavori d'artigiania degli hippy, come vere e proprie forme di lavoro. Per loro, il lavoro, rappresentava fatica, sudore, stanchezza accumulata dopo anni 
passati nei campi, e tutto ciò lo consideravano come un passatempo degli hippy. La credenza comune degli autoctoni, considerava gli hippy, come gente, che non amava lavorare, dei "fannulloni", perchè per loro non era concepibile che solo dopo due ore di lavoro, smettessero di lavorare per andare in spiaggia. Non capivano la filosofia degli hippy, avere soldi a sufficienza solo per vivere la giornata, così da avere più tempo da trascorrere con gli amici, in spiaggia, alle feste.

\subsection{Visioni opposte: droghe e sesso}

Non è possibile parlare di hippy, senza menzionare almeno questi due argomenti, le droghe ed il sesso. Molto spesso, il senso comune delle persone, relaziona ciò, alla cultura hippy. Si pensa che le droghe ed il sesso, facciano parte della vita quotidiana degli hippy ed è sempre opinione comune che le droghe allucinogene, come Lsd ed i suoi derivati, siano una cosa esclusiva degli hippy. Ma abbiamo visto che le cose sono leggermente diverse, e molte volte facciamo confusione tra il vero significato del termine hippy, e la nostra rappresentazione mentale comune a quello che pensa tutta la gente "normale".

L'acido lisergico, usato all'interno delle comunità hippy negli anni sessanta, è diversa da tutte le altre droghe, gli si attribuisce un significato del tutto differente, quasi come un rito da fare in gruppo.

E' molto differente dalla cultura delle droghe di oggi, sempre se di cultura è possibile parlare, in quanto oggi, l'assunzione delle droghe, è un fatto individuale, che lascia poco spazio alla comunità, al gruppo, allo stare insieme. Ora i discotecari che frequentano lbiza in estate, assumono ecstasy per riuscire a ballare tutta la notte, quasi come un integratore energetico, e ciò non viene percepito come un rito comune, ma come un'azione individuale.

Invece l'acido lisergico, era una droga che cambiava la percezione delle cose, cambiava la percezione della vita, alcuni la consideravano la droga dell'amore, dello stare insieme, tutto "Peace and Love", del "Fate l'amore, non fate la guerra".

Quando ci si relaziona con un'altra persona, la identità sessuale è la più rilevante.

Molti hippy vivevano insieme, con mogli e figli nella stessa casa, soprattutto questi figli erano nati fuori dal matrimonio, però gli autoctoni non facevano molto caso a ciò, la situazione cambiava, se era una ibicienca che aveva figli fuori dal matrimonio, in questo caso era uno scandalo.

Se per un giovane ibicienco era molto più facile relazionarsi con una ragazza hippy, per una ragazza ibicienca ciò era impossibile. Si racconta che in molte feste hippy, erano presenti giovani maschi autoctoni, e questi, attratti per le bellezze straniere, mai prima di allora viste, cercavano di conquistarle.

\section{CONCLUSIONI}

Il discorso fin qui fatto, appare svolgersi, contemporaneamente, su diversi livelli, che pur essendo dotati di regole e principi diversissimi, si intrecciano, si susseguono, si interpretano e si appoggiano gli uni sugli altri garantendo in questo modo il progresso della ricerca. Ogni capitolo descrive ed analizza diversi argomenti della Psicologia Sociale e delle scienze sociali, ma la lettura dell'intera tesi, garantisce omogeneità e linearità alla ricerca. 
Le due comunità ad Ibiza, hanno vissuto quel particolare periodo storico, gli anni sessanta, introducendo una nuova visione interculturale sull'isola. I comportamenti dei due gruppi, soprattutto la relazione endogruppo ed exogruppo, visti dalle due prospettive, quella degli hippy e quella degli autoctoni, ci dimostra come l'individuo ha una visione della società, molte volte, comune a quella degli altri. Possiamo notare come l'identità si costruisce attraverso un processo storico degli individui, gli autoctoni già erano un gruppo, ma gli hippy si sono costituiti in quell'epoca, in quelle circostanze sull'isola di Ibiza, l'identità degli hippy si è caratterizzata, ed è avvenuto il processo di creazione della comunità stessa. La comunità hippy si è creata in quel contesto, per differenziarsi dalla comunità locale. Con la creazione di queste due comunità, si è sviluppata l'identità sociale di ogni individuo appartenente al proprio gruppo. L'identità si è costituita in base al processo di comparazione tra il proprio gruppo ed il gruppo al quale l'individuo è alieno. Le differenze tra i due gruppi determinano l'identità sociale. Per gli hippy, la differenza con la comunità autoctona, consiste nel proprio stile di vita, come la concepiscono di forma opposta, la propria filosofia di base e l'apertura mentale verso l'altro, invece per gli autoctoni, la principale differenza con la comunità hippy è da ricercarsi nell'importanza che si da dei beni materiali, come ad esempio la casa ed il denaro, soprattutto la diversa concezione del lavoro, simbolo di progresso futuro. Possiamo essere d'accordo nell'affermare che la comunità autoctona è essenzialmente una comunità puramente interpersonale, che cioè il comportamento delle persone a cui appartengono, è determinato esclusivamente dalle caratteristiche individuali e le relazioni personali tra gli individui. La comunità hippy è di tipo intergruppale, in quanto tutta la condotta degli individui è quasi esclusivamente determinata per l'appartenenza delle persone allo stesso gruppo. Alla luce delle varie interviste, si coglie, una sorta di stereotipizzazione dell'io, in quanto le persone, sia hippy ed autoctoni, molte volte si percepiscono sé stessi come esemplari intercambiabili di una categoria sociale che come personalità uniche definite per le loro differenze individuali in relazione con gli altri.

Senza ombra di dubbio è l'aspetto discorsivista che ci interessa maggiormente della ricerca, infatti abbiamo notato ciò soprattutto incentrando l'analisi del contenuto, a proposito della comunicazione che avveniva tra le due comunità. Importantissima per capire com'è avvenuta l'integrazione delle due comunità. II processo d'integrazione è iniziato ad Ibiza per la prima volta, proprio in quel periodo, lo scambio interculturale tra le due comunità, avvenuto sotto il profilo discorsivista, della comunicazione quotidiana, per beni materiali. Pure la teoria delle Rappresentazioni Sociali fondata sull'azione, sulla comunicazione, costruita nel parlato e nel testo ci ha aiutato nell'analisi dei processi verbali, per definire il processo di scambio interculturale che è avvenuto sull'isola. La dinamica che scaturisce questa teoria è importante, per vedere come nella realtà, gli elementi che costituiscono i due gruppi, fanno parte di una rappresentazione collettiva, che risiede nella nostra mente, ed attraverso il linguaggio descriviamo queste rappresentazioni. Questo è particolarmente importante e significativo, perché ci aiuta a comprendere ed analizzare più precisamente le varie storie di vita. Captando queste particolari rappresentazioni, è possibile dimostrare come la dinamica discorsivista riesce sempre ad esplicare queste particolari descrizioni.

Le aspettative sono state ottenute, in luce di quanto scritto nella tesi. I risultati che abbiamo ottenuto ci sembrano interessanti se possono essere riferiti, a titolo di ipotesi generalizzabili, all'insieme dei discorsi nell'ambito delle scienze umane. Dovrebbero costituire inoltre, un certo numero di punti fermi che permettano una migliore comprensione delle procedure messe in opera nel corso dell'analisi contenutistica delle interviste. 
Pare dunque assodato che, l'integrazione tra ibicienchi ed hippy era dettata maggiormente dal rispetto per la diversità culturale, che di un vero e proprio adattamento comune delle due culture. Non c'è stato uno scambio reciproco comportamentale, di atteggiamenti, ma solo una più che rispettosa convivenza comune, dettata dal "lasciar vivere" e dai buoni propositi di entrambe le comunità. Esisteva una buona comunicazione tra le due culture, cosa che esiste tuttora, e che lascia presagire la tranquillità e serenità che abitava l'animo di entrambe le popolazioni.

\section{Referencias}

Alòs, A., Ask!? Ibiza\&Formentera, Factoria de Publicaciones, Barcellona, 2004.

Alvaro, J., Garrido, A., Psicología Social, McGraw-Hill, Madrid, 2003.

Baum P., La comuna, una alternativa a la familia, Ediciones Guadarrama, Madrid, 1975

Baum. Willa K, Oral History for the Local Historical Society, Nashville, American Association for State and Local History, 1987

Bohrnstedt G. W., Knoke D., Statistica per le scienze sociali, Bologna, II Mulino, 1998

Bruner J., La fabbrica delle storie, Bari, Laterza, 2002

Bruzzone, A., La cultura e il movimento hippie, Nuovi Accenti, Bologna, 2001.

Cabruja, T., Iñiguez, L., Vázquez, F., Cómo construimos el mundo: relativismo, espacios de relación y narratividad, Universitat Autónoma de Barcelona Servei de Publicacions, Barcellona, 2000.

Cashman, J.,El fenomeno LSD, Plaza \& Janes S.A., Barcellona, 1972.

Ceruti M., Bocchi G., Origini di storie, Feltrinelli, Milano, 1993.

Cirem Costa, F., Enciclopèdia d'Eivissa i Formentera, Industria grafica Bahia, Ibiza, 2000.

Corradi, C., Metodo biografico come metodo ermeneutico, Una rilettura de "II contadino polacco", Franco Angeli, Milano, 1988.

Crespo, E., Introducción a la Psicología Social, Editorial Universitas, Madrid, 1995

De Garay, G., "La entrevista de historia horal: monologo o conversación?" articolo tratto dalla rivista Revista Electrónica de Investigación Educativa, 1 (1), 1999, consultata il 23 marzo 2005 nel World Wide Web: http://redie.ens.uabc.mx/vol1no1/contendio-garay.html

De Grada,E., Bonaiuto; M., Introduzione alla psicologia sociale discorsiva, Editori Laterza, Bari, 2002.

Demazier D., Dubar C., Dentro le storie, Raffaello Cortina Editore, Milano,2000

De Villena, L.A., La revolución cultural (Desafío de una juventud), Editorial Planeta, Barcellona, 1975.

Dovigo F., (a cura di), Ciascun paese è mondo, Emi, Bologna, 2002

Dovigo F., Etnopedagogia, F. Angeli, Milano, 2002

Dovigo F., Osservazione e formazione, Angeli, Milano, 2003 
Garay, A., Iñiguez, L., Martinez, L., La perspectiva discursiva en Psicologia Social, articolo inviato alla rivista "Subjetividad y Procesos Cognitivos", Barcelona, 2003.

Folguera, P., Cómo se hace Historia Oral. Eudema, S.A. Madrid. 1994.

Gordo López, A. J., Linaza, J. L., Psicología, discursos y poder (PDP), Visor Dis., Madrid, 1996.

Greimas, A. J., Del Senso 2, Studi Bompiani, Milano, 1985.

Hannerz U., La diversità culturale, II Mulino, Bologna, 2001

Hebdige, D., Subculture- The meaning of style, Methuen, Londra, 1979.

Ibáñez Gracia, T., Aproximaciones a la Psicología Social, Sendai ediciones, Barcelona, 1990.

Ibáñez Gracia, T., Ideologías de la vida cotidiana, Sendai ediciones, Barcellona, 1988.

Ibáñez Gracia, T., Introducció a la Psicologia Social, Uoc, Barcelona, 2003.

Ibáñez Gracia, T., Iñiguez Rueda L., Critical Social Psychology, SAGE, Londra, 1997.

Ibáñez Gracia, T., Iñiguez Rueda L., Psicologia Social, Editorial UOC, Barcellona, 1996.

Iñiguez Rueda, L., Análisis del discurso, Editorial UOC, Barcellona, 2003.

Iñiguez Rueda, L., Antaki, C., El ànalisis del discurso en Psicologia Social, Boletin de Psicologia, Barcelona, 1994.

Ives, Edward D., The tape-Record Interview: A Manual for Field Workers in Folklore and Oral History, Knoxville, University of Tennessee Press, 1995.

Lepper, G., Categories in Text and Talk, Sage, Londra, 2000.

Lozano, J., Peña-Marín, C., Abril, G., Análisis del discurso, Catedra, Madrid, 1989.

Macabich, I., Historia de Ibiza IV, Daedalus, Palma di Mallorca, 1967.

Miles, B., Hippie, Cassell Illustrated, Londra, 2003.

Morales, J. F., Huici, C., Lecturas de Psicología Social, Mateu Cromo, Madrid, 1989.

Moscovici S. ( a cura di),La relazione con l'altro, Raffaello Cortina Editore,Milano,1997

Moscovici S.,Farr R. M. (a cura di),Rappresentazioni Sociali,Il Mulino, Bologna, 1989.

Partridge, W. L., The Hippie Ghetto The natural history of a subculture, Holt, Rinchart and Winston, New York, 1973.

Pujadas Muñoz, J.J., El método biográfico: El uso de las historias de vida en ciencias sociales, Centro de investigaciones sociológicas, Madrid, 1992.

Riera, J., Historia de Ibiza, Imprenta Alfa, Palma di Mallorca, 1954.

Ritchie, Donald A., Doing Oral History, New York, Twayne Publishers, 1995.

Ricolfi L. (a cura di), La ricerca qualitativa, Carocci, Roma, 1998. 
Romo, N., Mujeres y drogas de síntesis, Tercera Prensa-Hirugarren Prentsa, Donostia, 2001.

Salvador, L., Ibiza y Formentera, las baleares, La Foradada, Palma di Mallorca, 1984.

Schorzman, Terri A., A Practical Introduction to Videohistory: The Smithsonian Institution and the Sloan Foundation Experiment, Malabar, Krieger, 1993.

Stubbs, M., Análisis del discurso, Alianza Psicologia, Madrid, 1987.

Tajfel H., Gruppi umani e categorie sociali, II Mulino, Bologna, 1995.

Turner, J. C., Rediscovering the social group. A self-categorization theory, Basil Blackwell Ltd., Oxford, 1987.

Van Dijk, T., El discurso como estructura y proceso, Gedisa editorial, Barcellona, 2000.

Van Dijk, T., El discurso como interacción social, Gedisa Editorial, Barcellona 2001.

Van Dijk, T., Estructuras y funciones del discurso, Siglo Veintiuno editores, Cittá del Messico, 1980.

Vázquez, F., La memoria como acción social, Paidós Ibérica, Barcelona, 2001.

Vázquez, F., Objetivos y medios en la investigación psicosocial, Università Autonoma di Barcelona, Barcelona, 1996

Vila-Matas, E., Bartleby y compañeros, Anagrama, Madrid, 2003

\section{Formato de citación}

Belli, Simone (2005). Integrazione sociale in comunità rurali: il caso concreto della comunità hippy in Ibiza. Athenea Digital, 8. Disponible en http://antalya.uab.es/athenea/num8/belli.pdf

\section{SOLERIGHIS RESERVED}

Este texto está protegido por una licencia Creative Commons.

Usted es libre de copiar, distribuir y comunicar públicamente la obra bajo las siguientes condiciones:

Reconocimiento: Debe reconocer y citar al autor original.

No comercial. No puede utilizar esta obra para fines comerciales.

Sin obras derivadas. No se puede alterar, transformar, o generar una obra derivada a partir de esta obra.

$\underline{\text { Resumen de licencia }}$

$\underline{T e x t o ~ c o m p l e t o ~ d e ~ l a ~ l i c e n c i a ~}$ 